\title{
Los modelos de interacción espacial y el análisis de los flujos migratorios interregionales. Aplicación al territorio español
}

José Miguel SANTOS

Las complejas relaciones sociales y económicas de las sociedades postindustriales han transformado el modelo tradicional de la organización territorial. Los avances en el campo de la informática y las telecomunicaciones y el desarrollo de los medios de transporte han hecho posible la existencia simultánea de dos hechos aparentemente contrapuestos: la concentración de la actividad humana en gigantescas aglomeraciones urbanas y la dispersión geográfica de la actividad económica. Ambas tendencias aparecen presentes en el mundo actual. La internacionalización de la economía y las modernas tecnologias han representado un factor determinante en la deslocalización de las actividades empresariales en el mundo capitalista. Mientras, de forma paralela, el crecimiento de los mercados financieros y comerciales internacionales ha favorecido el control centralizado y la dirección ejecutiva de la economía y en consecuencia la concentración de los servicios de gestión de alto nivel en las grandes ciudades. No es de extrañar, que «la transformación espacial y técnica de la actividad económica incluya la dispersión geográfica de fábricas, oficinas y mercados de servicios, así como un fuerte incremento del uso de servicios altamente especializados, frecuentemente ligados al desarrollo de la microelectrónica. Ambos procesos, dispersión y especialización de servicios, son interactivos y superpuestos» (SASSEN, S., 1992).

Esta nueva forma de organización espacial de la actividad humana, en sus dos niveles de escala: interurbano e intraurbano, ha incrementado los flujos de relación originados por los diferentes subsistemas de actividad. La gran ciudad se ha convertido, hoy en día, en una compleja estructura, integrada por un amplio mosaico de usos del suelo, dispersos por un territorio que supera a veces los cincuenta kilómetros de radio. Es natural, 
por tanto, que cualquiera de las actividades diarias de sus habitantes, por diversos motivos de trabajo, ocio, utilización de múltiples servicios, etc, originen importantes movimientos de personas, bienes e ideas en el espacio. $Y$ qué decir al nivel de la escala que traspasa los límites de la ciudad. Actualmente, los flujos de relación interurbanos superan con facilidad las fronteras nacionales en un contexto de creciente internacionalización de la economía.

Estas circunstancias explican el auge espectacular experimentado por los modelos de interacción espacial por dar respuesta -explicativa e incluso predictiva - a alguno de los principales problemas planteados en el ámbito de la ciencia regional. Las formulaciones matemáticas implícitas en este tipo de modelos han sido aplicadas con éxito en diversas áreas del conocimiento geográfico. En el campo de la demografía, el análisis de las migraciones interregionales o interurbanas ha permitido identificar los determinantes básicos, relativos a la atracción de los lugares de destino o rechazo de los lugares de origen, que explican la diferenciación espacial de los flujos característicos de este tipo de movimiento poblacional. Asimismo, los modelos interactivos han sido aplicados al estudio de las redes de transporte o a la localización óptima de los servicios en la ciudad, desde diversos planteamientos teóricos de minimización de costes y distancias.

\section{LOS MODELOS DE INTERACCIÓN ESPACIAL}

La interacción espacial puede ser definida "como las relaciones de movimiento - de personas y bienes - y comunicación - de ideas e información-que tienen lugar en el espacio, como consecuencia de un complejo proceso de toma de decisiones» (Fotheringhan, A. S., Y O'KelLy, M. E., 1987). El término puede ser aplicado en diversos campos de la ciencia regional, donde el comportamiento de los individuos o grupos sociales sea decisivo en la magnitud alcanzada por determinados movimientos o flujos interactivos: migraciones interregionales, movimientos diarios de commuters, viajes por motivo de recreo o elección de un determinado servicio público, llamadas de teléfono, movimientos de capitales, etc.

Todo sistema de interacción espacial precisa la definición de sus elementos o componentes, de los atributos o propiedades de éstos y de las relaciones de interdependencia o acoplamiento mutuo (MURCIA, E., 1979). A modo de ejemplo, si nos refiriéramos a la interacción espacial en el interior de una ciudad, por motivos de ocio y recreo (asistencia a espectáculos, restaurantes, etc.), el mosaico de áreas residenciales contituiría la estructura espacial de los elementos emisores, mientras que las áreas especializadas 
en la localización de las funciones recreativas serian los elementos receptores. En el resultado del conjunto del sistema: flujos de personas que se desplazan por el motivo considerado, tienen gran importancia las propiedades o características de los elementos emisores: atributos de la demanda (nivel social, capacidad económica, preferencias y gustos, etc.), así como las de los elementos receptores: atributos de la oferta (características y localización relativa de los bienes ofertados).

En otro nivel de escala, los movimientos migratorios pueden ser considerados como el resultado de la interacción espacial entre las regiones de un pais. En este nuevo ejemplo concreto, los componentes receptores y emisores serian los mismos: las unidades administrativas regionales, (variando, únicamente, su carácter, como foco generador de movimiento o como foco de atracción). La magnitud de los flujos migratorios interactivos dependería de las propiedades de los lugares de origen (nivel de subdesarrollo, expectativas de cambio, posible situación de crisis económica, etc.) y de los atributos de los lugares de recepción (oferta de empleo, mejora de las condiciones de vida, etc.).

\section{El modelo matemático de la interacción espacial}

El origen de cualquier modelo de interacción espacial es el resultado del sistema: una matriz rectangular de flujos, entre los «m» lugares de origen y los «n» lugares de destino:

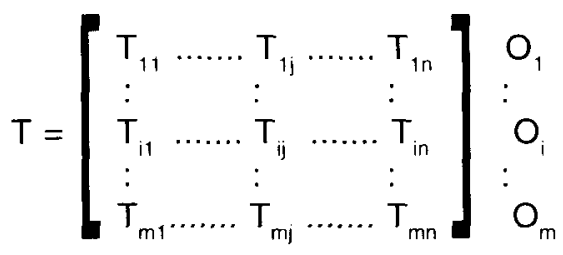

$$
\begin{aligned}
& D_{1} \ldots \ldots D_{i} \ldots \ldots D_{n} \\
& O_{i}=\sum_{j=i}^{n} T_{i j} \quad D_{i}=\sum_{i=1}^{m} T_{i j}
\end{aligned}
$$

La suma de interacciones $\mathrm{O}_{i}$, correspondiente a una fila, supone el flujo total emitido por cada lugar de origen, mientras que la suma de interaccion $D_{i}$, correspondiente a una columna, representaría el flujo total recibido en cada lugar de destino. 
Los modelos de interacción espacial implican que los valores de los flujos $T_{i j}$, entre un elemento emisor «i” y un elemento receptor " $j$ ", son la consecuencia de tres tipos de factores:

a) El primero está relacionado con la fricción espacial. Parece lógico suponer que la mayor o menor dificultad en establecer la relación, como consecuencia de la localización relativa en el espacio, sea determinante en el valor de la interacción. La forma de medir la resistencia del espacio al movimiento puede ser variada: el coste de desplazamiento, el tiempo de desplazamiento o la distancia. De los tres tipos de medida, la última es la más utilizada.

La distancia puede ser obtenida si conocemos las coordenadas de los puntos de origen $\left(x_{i}, y_{i}\right)$ y de los puntos de destino $\left(x_{i}, y_{i}\right)$, de forma general, mediante la fórmula:

$$
d_{i j}=K\left[\left(x_{i}-x_{j}\right)^{p}+\left(y_{i}-y_{j}\right)^{p}\right]^{\frac{1}{p}}
$$

Para el valor $p=2$, obtendriamos la distancia euclideana:

$$
d_{i j}=K \sqrt{\left(X_{i}-X_{j}\right)^{2}+\left(y_{i}-Y_{j}\right)^{2}}
$$

mientras que el valor $p=1$, daría como resultado la distancia rectangular:

$$
d_{i j}=k\left[\left(x_{i}-x_{j}\right)+\left(y_{i}-y_{j}\right)\right]
$$

La distancia euclideana representa la distancia en línea recta y se adecua mejor al nivel de la escala interurbana, mientras que la distancia rectangular to hace en el nivel de la escala intraurbana.

De forma general, la matriz $C$ contendria los valores $C_{i j}$ de fricción espacial existentes entre cada par de elementos interactivos, « $j » y$ « $j »$ :

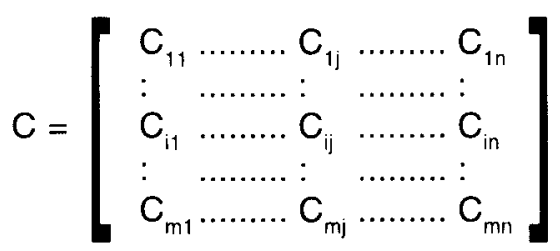

b) Un segundo tipo de variables está relacionado con las características o atributos de los lugares de origen y su capacidad para impulsar el movimiento. Si continuamos refiriéndonos al ejemplo de los movimientos migratorios, este tipo de variables representaría el rechazo del medio y 
su papel determinante en la toma de decisión de emigrar. Si suponemos la influencia de «p» factores, la matriz V estaría integrada por los valores de los mismos, correspondientes a los «m» lugares de origen:

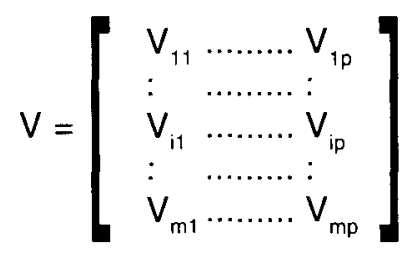

c) Finalmente, el tercer tipo de variables estaría relacionado con los factores de atracción de los lugares de destino. En el caso de los movimientos migratorios, este tipo de variables representaría el nivel de desarrollo de los elementos de recepción y su capacidad de influir en el proceso de toma de decisiones del cambio permanente de residencia. Si suponemos la influencia decisiva de " $q$ " factores, la matriz $W$ estaria integrada por los valores de los mismos, correspondientes a los «n» lugares de destino:

$$
W=\left[\begin{array}{lllc}
W_{11} \ldots & W_{1 j} \ldots & W_{1 n} \\
\vdots & \vdots & & \vdots \\
\vdots & \vdots & & \vdots \\
W_{q 1} \ldots & W_{q j} \ldots & W_{q n}
\end{array}\right]
$$

La expresión más general de la formalización matemática existente entre los flujos interactivos $T_{i j}$ y los factores determinantes de la misma $C_{i j}, V_{i}$ y $W_{i}$ sería:

$$
T_{i 1}=f\left(\alpha_{1} V_{i 1}, \alpha_{2} V_{i 2}, \ldots \ldots, \alpha_{p} V_{i p} ; B_{1} W_{1 i}, B_{2} W_{2 j}, \ldots \ldots, B_{q} W_{q j} ; \gamma C_{i j}\right)
$$

siendo $\alpha, \beta$ y $\gamma$ los parámetros que reflejan la relación.

La estructura del modelo puede simplificarse si asumimos la existencla de un único factor de impulsión $(p=1)$ y de atracción $(q=1)$ :

$$
T_{i j}=f\left(\alpha V_{i} ; \beta W_{j} ; \gamma C_{i j}\right)
$$

La relación funcional puede ser diferente para cada tipo de variable. 
Está bastante generalizada la opinión de que los factores $v_{i}$ y $w_{j}$ se ajustan mejor a funciones de carácter potencial, mientras que la fricción espacial $\mathrm{C}_{\mathrm{ij}}$ lo hace tanto con este tipo de funciones, como exponencial:

$$
\begin{aligned}
& f\left(\alpha V_{i}\right)=V_{i}^{\alpha} \\
& f\left(\beta W_{j}\right)=W_{i}^{\beta} \\
& f\left(\gamma C_{i j}\right)=C_{i j}^{\gamma} \quad o \quad e^{\gamma C_{i j}}
\end{aligned}
$$

2. Tipos de modelos. Técnicas de minimización de la información

Los modelos matemáticos se elaboran, en general, con la finalidad de formalizar las relaciones existentes en el mundo real. Dos objetivos fundamentales están en la base de su utilización: la explicación y la predicción. Refiriéndonos a los modelos de interacción espacial, el carácter explicativo de los mismos reside en la estimación o calibración de los parámetros, que permita una más adecuada correspondencia de la información empírica respecto a la formulación teórica del modelo. Por su parte, la utilización de los modelos interactivos con carácter predictivo plantea la deducción de los flujos de interrelación a partir de factores de impulsión o atracción ya conocidos.

En el campo de la explicación, tres tipos de modelos diferentes existen. En primer lugar, podemos destacar los denominados «no restrictivos», que presuponen el conocimiento de los atributos de los elementos de origen y de destino. Junto a ellos, se encontrarían los modelos con «restricción de origen" y "restricción de destino" según poseyéramos información exclusiva de las características de los elementos impulsores o de atracción.

El cuarto tipo de modelo: "doblemente restrictivo» contiene un propósito fundamentalmente predictivo y busca obtener, precisamente, los flujos de correspondencia existentes entre los lugares de origen y destino.

Las relaciones inherentes a los cuatro tipos de modelos descritos son las siguientes:

a) No restrictivos. Ni el conjunto de los totales de las filas $\mathrm{O}_{i}$, ni el de las columnas $\mathrm{D}_{\mathrm{j}}$ es conocido.

b) Restricción de origen. El conjunto de los totales de las filas $\mathrm{O}_{i}$ es conocido.

c) Restricción de destino. El conjunto de los totales de las columnas $D_{j}$ es conocido. 
d) Doblemente restrictivo. Ambos totales (de filas y columnas) $\mathrm{O}_{i}$ y $\mathrm{D}_{\mathrm{j}}$ son conocidos.

Los modelos elaborados y actualmente en uso contienen las restricciones ya apuntadas, a la par que introducen conceptos de optimización en la línea de la maximización de la entropía (Shannon, C. F., 1948) y/o minimización de la información (EvaNs, R. A., 1969). El resultado son distintas familias de modelos matemáticos cuya formulación expresamos a continuación:

Modelos no restrictivos

$$
T_{i j}=V_{i}^{\alpha} W_{j}^{\beta} \exp \left(-\gamma d_{i j}\right)
$$

Modelos de restricción de origen

$$
\begin{aligned}
T_{i i}=A_{i} O_{i} \exp \left(-\gamma d_{i j}\right) \\
\text { siendo } \quad A_{i}=\frac{1}{\sum_{i} \exp \left(-\gamma d_{i j}\right)}
\end{aligned}
$$

Modelos de restricción de destino

$$
\begin{aligned}
T_{i j}=B_{j} D_{j} \exp \left(-\gamma d_{i j}\right) \\
\text { siendo } \quad B_{j}=\frac{1}{\sum_{j} \exp \left(-\gamma d_{i j}\right)}
\end{aligned}
$$

Modelos doblemente restrictivos

$$
\begin{gathered}
T_{i j}=A_{i} B_{j} O_{i} D_{j} \exp \left(-\gamma d_{i j}\right) \\
\text { siendo } A_{i}=\frac{1}{\sum_{i} B_{i} D_{j} \exp \left(-\gamma d_{i j}\right)} \\
B_{j}=\frac{1}{\sum_{i} A_{i} O_{i} \exp \left(-\gamma d_{i j}\right)}
\end{gathered}
$$




\section{LA APLICACIÓN DE LOS MODELOS DE INTERACCIÓN ESPACIAL AL ESTUDIO DE LOS MOVIMIENTOS MIGRATORIOS INITERREGIONALES EN ESPAÑA}

\section{Las nuevas tendencias migratorias de los paises desarrollados}

En la mayoría de los países, la redistribución espacial de la población, como consecuencia de los movimientos migratorios, ha sido un factor determinante de los desequilibrios territoriales. La concentración en las áreas urbanas de la población joven y más cualificada profesionalmente ha generado un proceso de desarrollo selectivo, que ha beneficiado determinadas regiones en perjuicio de otras. Hasta hace poco tiempo, las grandes metrópolis de los países desarrollados, áreas prioritarias de la concentración de los medios productivos y el empleo, se habían convertido en los lugares privilegiados del crecimiento económico. Los flujos netos de migración hacia estas áreas habían producido un efecto multiplicador sobre el empleo, provocando, paralelamente, la decadencia de las áreas rurales.

Sin embargo, la crisis económica que desde mediados de los años setenta viene afectando, con altibajos, a los países desarrollados ha tenido importantes consecuencias sobre el tradicional modelo territorial urbano, que ha supuesto, en parte, la quiebra del modelo de concentración de la población y los recursos en las grandes aglomeraciones urbanas. Nos encontramos, por tanto, en un momento histórico insólito, en el que se corta un largo proceso de concentración que contaba con siglos de antigüedad. Las causas de este fenómeno hay que buscarlas en una inversión de los procesos de polarización, como consecuencia de la reorganización de la actividad productiva. La causa del proceso descentralizador de la industria, bien hacia la periferia de las grandes ciudades, bien hacia ciudades de menor tamaño del propio país, o incluso hacia paises tercermundistas hay que buscarla en un variado conjunto de factores productivos. La elevación del precio de la energía y las materias primas, el aumento de los costes directos (salarios) e indirectos (prestaciones sociales) del trabajo, la elevación de los costes financieros y de la presión fiscal para las empresas, el aumento de la competencia en ciertas ramas por la incorporación de nuevos países o la rápida innovación tecnológica son algunas de las causas principales de la desaceleración del crecimiento de la industria tradicional de la gran ciudad. Las consecuencias más visibles han sido el cierre de empresas, el reajuste de plantillas de las industrias de mayor tamaño y su reorganización a una escala más reducida, en condiciones de restablecer la rentabilidad económica. 
Si bien este fenómeno ha sido bastante generalizado, nuevas funciones urbanas han revitalizado el papel estratégico de las grandes ciudades. La consideracion de constituir centros de poder altamente concentrado en la organización de la economía mundial, la localización de las empresas financieras y de servicios especializados y el servir de lugares productores de innovación (SASSEN, S., 1991) han reestructurado un nuevo orden económico y social urbano. Sin embargo, los datos censales son bastante reveladores del declive urbano. En todo el mundo industrial desarrollado, cambios profundos están alterando las pautas geográficas de la residencia y el trabajo. La difusión de determinadas actividades productivas sobre el territorio ha desacelerado, y en algunos casos invertido, la tradicional sangría de determinadas regiones, causada por el éxodo migratorio. Según Campos Venuffi (1985), el modelo de crecimiento cuantitativo habría entrado en crisis, provocando la quiebra de los mecanismos que inducian los desequilibrios territoriales.

En nuestro país, el diagnóstico definitivo está aún por venir. Por una parte, "el proceso general de desindustrialización ha alcanzado de lleno a las áreas urbanas, y en particular a aquéllas con una mayor tradición industrial" (QUERO CASTANYS, D., 1985). Este fenómeno se ha producido, paralelamente, a ciertos procesos autonómos de industrialización del medio local, que simplemente por partir de unos niveles de deterioro máximo, parecen haber mejorado su situación relativa. Sin embargo, "el proceso de recuperación económica dominante en la segunda mitad de los años ochenta, una vez superada la fase inicial de la reestructuración, elevó de nuevo la cifra de establecimientos y empleos industriales en los espacios metropolitanos" (MÉndez, R., y CaRAVACA, 1., 1993), dejando pendiente la afirmación de hasta qué punto se trata de tendencias estructrurales o meramente circunstanciales. Además, el importante peso relativo del sector servicios en la economía española y el aumento de la participación del sector terciario en las grandes regiones metropolitanas ha frenado, en parte, las tendencias migratorias negativas previstas según el modelo general.

Sin embargo, podemos afirmar el profundo cambio experimentado en las pautas demográficas del espacio geográfico español. Los considerables aportes migratorios que provocaron el acelerado crecimiento demográfico de determinadas regiones (Madrid, Cataluña, País Vasco, etc.), antes de 1975, han quedado reducidos a su mínima expresión, siendo en algunos casos, incluso negativos. Los saldos migratorios netos se han reducido sensiblemente, aunque encubren, en realidad, importantes movimientos migratorios entre regiones. 
2. La aplicación de los modelos de interacción espacial al análisis de los movimientos migratorios

El objetivo de nuestra investigación tiene un carácter fundamentalmente explicativo y no predictivo. Se trata de aplicar el modelo de interacción espacial a las matrices de flujos migratorios interregionales (comunidades autónomas), habidas en nuestro país, en dos momentos claramente diferenciados en su dinámica demográfico-territorial: el periodo del desarrollismo económico (año 1970) y el momento actual (año 1990).

Con este planteamiento, intentamos valorar la influencia diferencial en el tiempo de los diversos tipos de factores que contribuyen decisivamente a determinar la magnitud de los movimientos migratorios a escala regional: la fricción del espacio, la jerarquía poblacional y los factores de atracción $y$ rechazo de los lugares de origen y destino.

\subsection{La base empirica. La medida de las principales variables explicativas del modelo de interacción espacial}

El primer paso de la investigación ha consistido en la recogida y tranformación de la información empírica precisa para la aplicación del modelo de interrelación espacial. Los componentes del sistema interactivo migratorio han sido las diecisiete comunidades autónomas, tal como han quedado definidas tras la nueva reorganización administrativa del territorio español. Las variables que necesitábamos para la validación del modelo general, referidas a los años 1970 y 1990, eran las siguientes:

- Matriz de flujos migratorios anuales entre comunidades autónomas.

- Matriz de distancias euclideanas relativas, entre las mismas unidades.

- Vectores de información de determinadas variables o atributos, representativos de la atracción de los lugares de destino o de la impulsión desde los lugares de origen.

a) Los valores correspondientes a la matriz de los flujos migratorios del año 1990 (cuadro II) se han obtenido directamente del Anuario Estadístico del INE, relativo al año de referencia.

La fuente de información del año 1970 ha sido la misma, aunque hemos debido comprimir la matriz de flujos migratorios entre las provincias españolas (de dimensión $50 \times 50$ ), en otra más reducida, relativa a las unidades de la escala regional actual (cuadro I). 
b) El cálculo de las distancias existentes entre las comunidades autónomas presentaba un mayor nivel de complejidad. El problema lo hemos resuelto en dos fases diferenciadas:

- Definición y cálculo de las coordenadas geográficas de los centros de gravedad de cada una de las diecisiete unidades territoriales.

- Cálculo de la matriz de distancias existente entre cada dos de estos elementos (matriz cuadrada y simétrica de $17 \times 17$, con los valores de la diagonal nulos).

- El cálculo del centro de gravedad del territorio delimitado por cada comunidad autónoma lo hemos obtenido de forma ponderada (por el volumen de población), a partir de las coordenadas geográficas de las provincias que lo integran, suponiendo la población total provincial concentrada en cada una de las capitales provinciales:

$$
\begin{aligned}
& \bar{X}=\frac{\sum X p}{\sum p} \\
& \bar{Y}=\frac{\sum Y p}{p}
\end{aligned}
$$

siendo $\bar{X}$ e $\bar{Y}$ las coordenadas del centro de gravedad del territorio regional y $X, Y$ y $p$ las coordenadas y población de cada unidad provincial.

Los resultados han sido lógicamente diferentes para los años 1970 y 1990, como consecuencia de la variación relativa de la distribución espacial de la población.

- En una fase posterior, hemos obtenido las distancias relativas entre cada dos puntos (definidos por sus coordenadas geográficas de longitud y latitud), resolviendo el triángulo esférico delimitado por ambos y el Polo Norte (figura 1).

En cada triángulo esférico, conoceríamos los valores de dos lados «b» y " $C$ " (colatitudes de los lugares de referencia) y del ángulo diedro (diferencia de los valores de las longitudes). Se trataría de determinar el valor del tercer lado "a» y traducir la distancia en grados a su valor correspondiente en unidades de longitud.

A modo de ejemplo, procedemos a exponer el procedimiento de cálculo aplicado a dos cualesquiera de las comunidades autónomas (Madrid y Cataluña) (año 1990). 


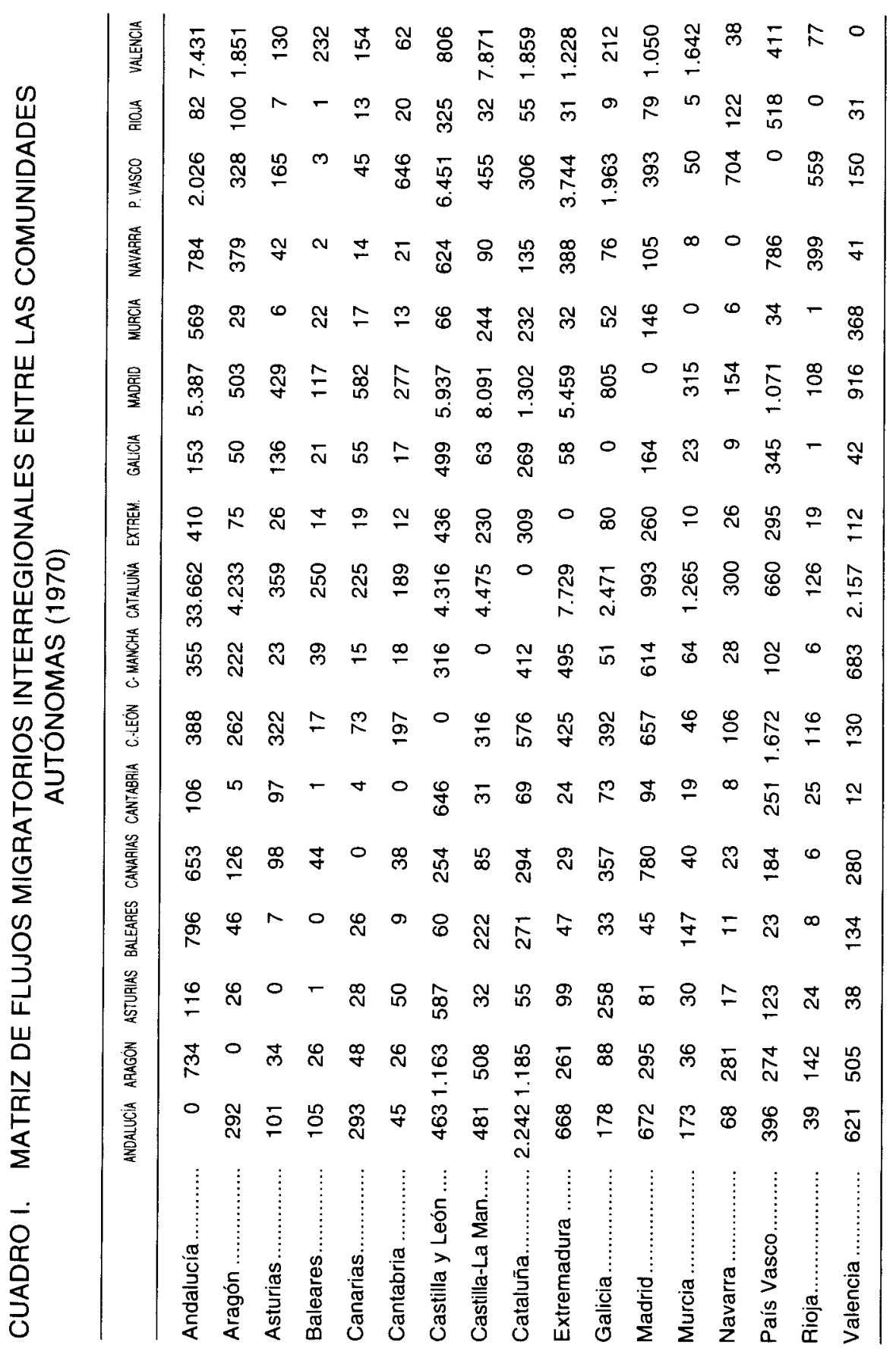




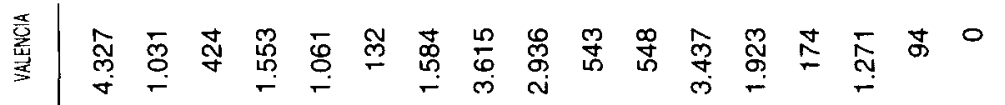

吉

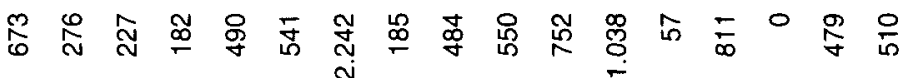

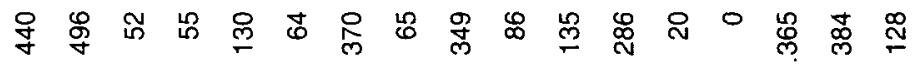

罗

․ ल ल N 0 ब

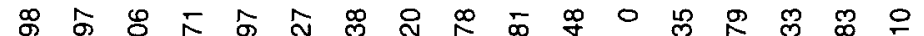

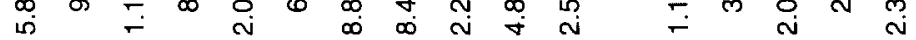

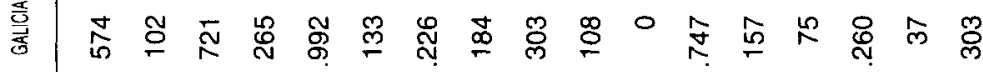

๘

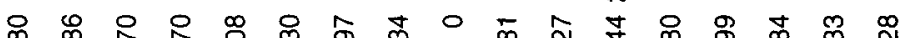
市 풍 吉 䒚 m

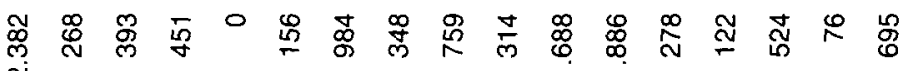

恖

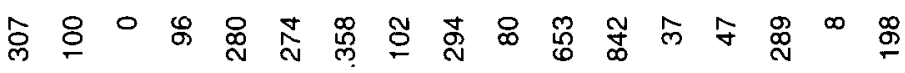

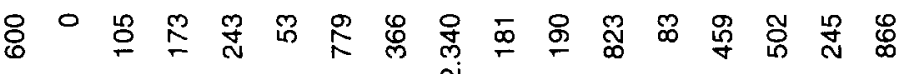




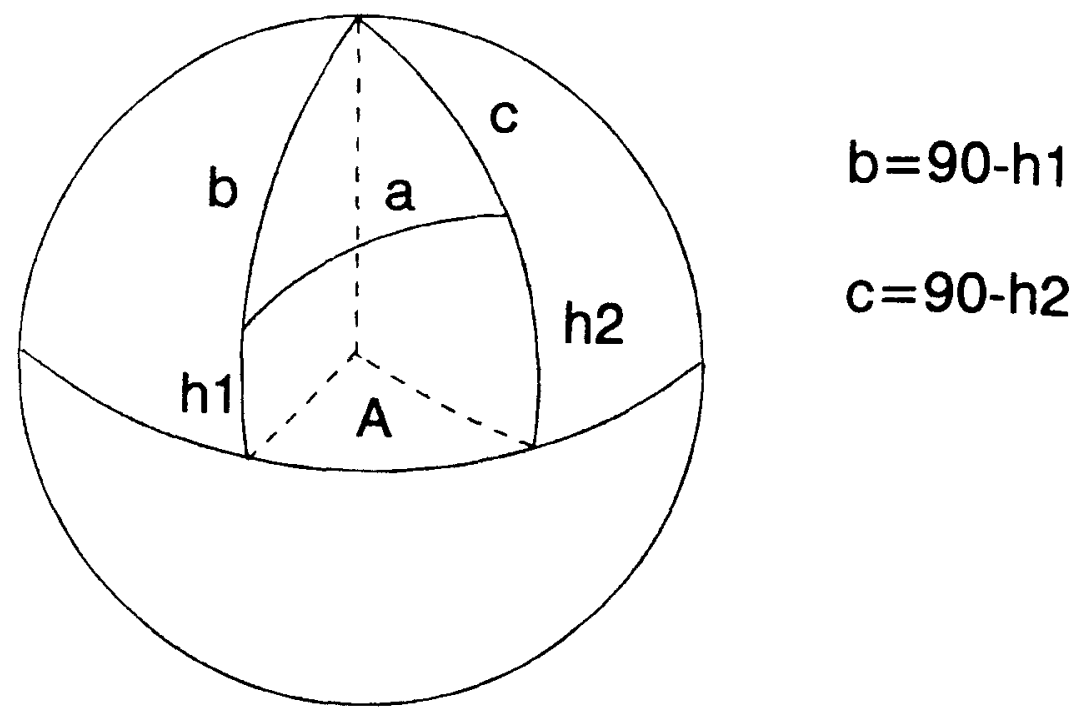

Fig. 1. Cálculo de la distancia métrica entre dos puntos de la superficie terrestre de latitudes $h_{1}$ y $h_{2}$ y diferencia de longitudes $A$.

Los valores de los lados b y c y el ángulo $A$ serían:

$\mathrm{b}=90-40,42=49,58^{\circ} \quad\left(40,42^{\circ}\right.$ latitud de Madrid $)$

$\mathrm{C}=90-41,44=48,56^{\circ} \quad\left(41,44^{\circ}\right.$ latitud de Cataluña $)$

$A=5,71-0=5,71^{\circ} \quad\left(0^{\circ}\right.$ y $5,71^{\circ}$ Iongitudes de Madrid y Cataluña $)$

Resolviendo el triángulo esférico:

$$
\begin{gathered}
\operatorname{tg} \frac{B+C}{2}=\frac{\cos \frac{b-c}{2}}{\cos \frac{b+c}{2} \operatorname{tg} \frac{A}{2}}=\frac{\cos 0,51^{\circ}}{\cos 49,07^{\circ} \operatorname{tg} 2,85^{\circ}}=30,61 \\
\operatorname{tg} \frac{B-C}{2}=\frac{\operatorname{sen} \frac{b-c}{2}}{\operatorname{sen} \frac{b+c}{2} \operatorname{tg} \frac{A}{2}}=\frac{\operatorname{sen} 0,51^{\circ}}{\operatorname{sen} 49,07^{\circ} \operatorname{tg} 2,85^{\circ}}=0,24 \\
\frac{B+C}{2}=88,13^{\circ}
\end{gathered}
$$




$$
\begin{gathered}
\frac{B-C}{2}=13,50^{\circ} \\
\operatorname{tg} \frac{a}{2}=\frac{\operatorname{tg} \frac{b-C}{2} \operatorname{sen} \frac{B+C}{2}}{\operatorname{sen} \frac{B-C}{2}}=\frac{\operatorname{tg} 0,51^{\circ} \operatorname{sen} 88,13^{\circ}}{\operatorname{sen} 13,50^{\circ}}=0,03865 \\
\frac{a}{2}=2,2136^{\circ} \\
a=4,4273^{\circ}=4,4273 * 111,11\left(\mathrm{~km} / 1^{\circ}\right)=491,92 \mathrm{~km}
\end{gathered}
$$

Un cálculo similar, para el resto de las combinaciones posibles entre las diecisiete comunidades autónomas, nos daría las matrices de distancias relativas a los años 1970 y 1990 (cuadros III y IV).

c) Finalmente, hemos seleccionado la información de algunas variables que consideramos han podido influir decisivamente en la magnitud de los flujos migratorios interregionales: la jerarquia urbana (medida por el volumen de la población), los factores de rechazo de los lugares de origen (medidos por el porcentaje de población activa agraria) y los factores de atracción de los lugares de destino (medidos por el porcentaje de la población industrial y nivel de los salarios) (cuadro V).

\subsection{La capacidad explicativa de los modelos de interacción espacial.}

\section{La aplicación del modelo no restrictivo}

La aplicación del marco teórico de la interacción espacial al estudio de los movimientos migratorios ha estado orientada, desde los tres tipos de modelos fundamentales: "no restrictivos", "restrictivos" (de origen o de destino) y "doblemente restrictivos". El primero de ellos ha sido empleado en numerosos análisis e investigaciones (CLARK, G. L., y BALLARD, K. P., 1980; Lovett, A. A., Whyte, I. D., y White, K. A., 1985; Flowerdew, R., y SALT, J., 1979; etc.) con el objeto de explicar el papel desempeñado por los atributos de los lugares de impulsión y atracción en el resultado final de los flujos migratorios.

Este modelo, como ya adelantamos en alguno de los apartados precedentes, presenta la siguiente formulación matemática:

$$
T_{i j}=K V_{i}^{\alpha} W_{j}^{\beta} C_{i j}^{\gamma}
$$




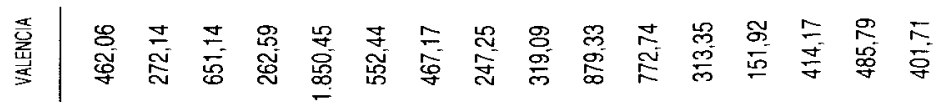

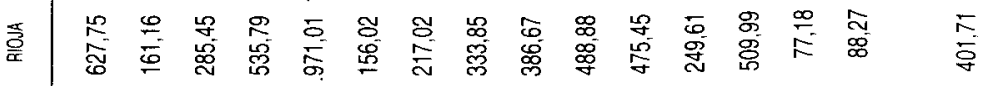

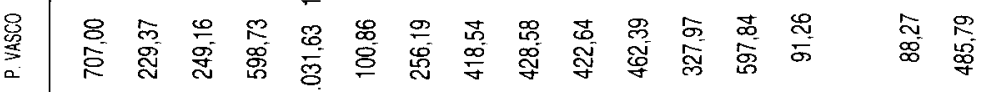
焉

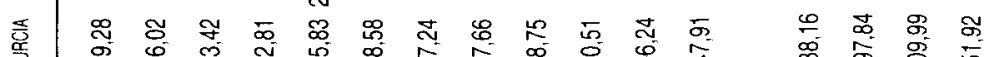

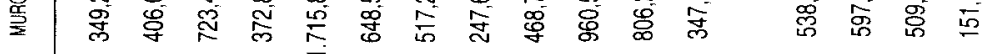

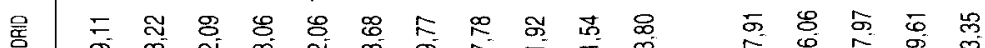

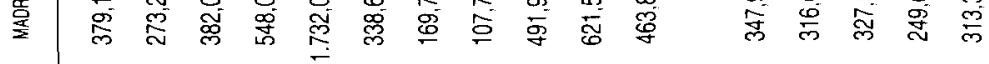
늘

투

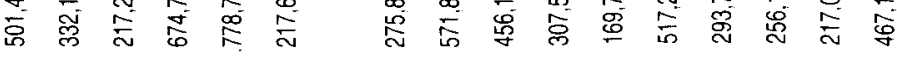
-

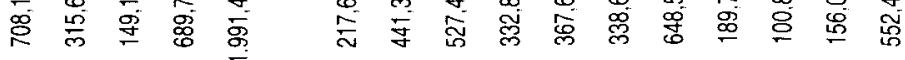
范

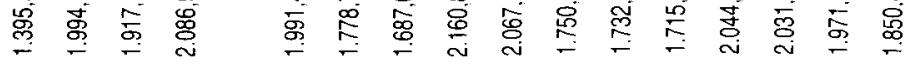

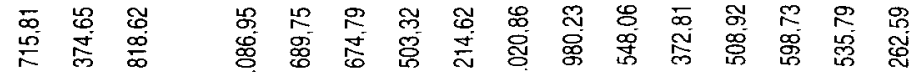
$\frac{N}{\mathbb{I}}$ म

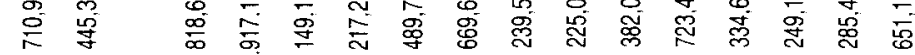
$\infty$ m

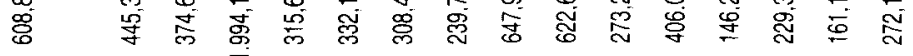
-

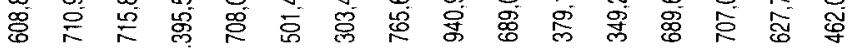

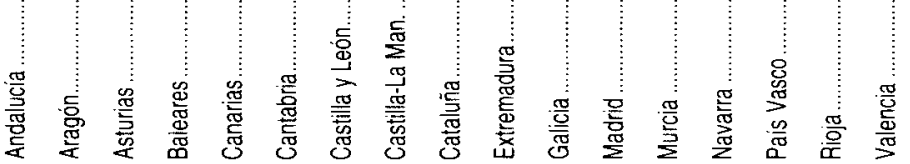







CUADRO V. ATRIBUTOS DE LOS LUGARES DE ORIGEN Y DESTINO, ESTIMADOS COMO CAUSA FUNDAMENTAL DE LOS FLUJOS MIGRATORIOS (1970 Y 1990)

\begin{tabular}{|c|c|c|c|c|c|c|c|}
\hline \multirow[b]{2}{*}{$\begin{array}{c}\text { AMBITO } \\
\text { TERRITORIAL }\end{array}$} & \multicolumn{3}{|c|}{1970} & \multicolumn{4}{|c|}{1990} \\
\hline & $\begin{array}{l}\text { VOLUMEN } \\
\text { DE } \\
\text { POBLACION }\end{array}$ & $\begin{array}{l}\text { PORCENTAJE } \\
\text { POBLACIÓN } \\
\text { AGRARIA }\end{array}$ & $\begin{array}{c}\text { PORCENTAJE } \\
\text { POBLACIÓN } \\
\text { INDUSTRIAL } \\
\text { (METAL) }\end{array}$ & $\begin{array}{l}\text { VOLUMEN } \\
\text { DE } \\
\text { POBLACIÓN }\end{array}$ & $\begin{array}{l}\text { PORCENTAJE } \\
\text { POBLACIÓN } \\
\text { AGRARIA }\end{array}$ & $\begin{array}{c}\text { PORCENTAJE } \\
\text { POBLACIÓN } \\
\text { INDUSTRIAL }\end{array}$ & $\begin{array}{c}\text { NIVEL } \\
\text { DE } \\
\text { SALARIOS }\end{array}$ \\
\hline Andalucia & 5.991 .076 & 45,50 & 1,91 & 6.919 .750 & 13,29 & 14,47 & 1.002 \\
\hline Aragón & 1.153 .055 & 29,13 & 4,60 & 1.212 .524 & 12,50 & 24,81 & 1.129 \\
\hline Asturias & 1.052 .048 & 21,63 & 1,84 & 1.125 .949 & 14,81 & 21,16 & 1.190 \\
\hline Baleares & 532.946 & 17,25 & 0,76 & 682.371 & 4,79 & 16,48 & 966 \\
\hline Canarias & 1.125 .442 & 24,67 & 0,43 & 1.484 .745 & 8,89 & 8,96 & 890 \\
\hline Cantabria & 469.077 & 27,53 & 3,78 & 527.173 & 11,64 & 21,51 & 1.099 \\
\hline Cast.-León & 2.668 .284 & 38,45 & 1,90 & 2.625 .751 & 17,01 & 19,06 & 1.083 \\
\hline Cast.-La Man. & 1.732 .696 & 42,47 & 0,56 & 1.713 .529 & 15,89 & 23,50 & 855 \\
\hline Cataluña & 5.107 .606 & 8,41 & 8,41 & 6.007 .622 & 3,62 & 31,96 & 1.116 \\
\hline Extremadura & 1.169 .396 & 49,20 & 0,40 & 1.128 .039 & 21,06 & 9,59 & 905 \\
\hline Galicia & 2.676 .403 & 48,99 & 2,14 & 2.804 .200 & 29,73 & 15,80 & 941 \\
\hline Madrid & 3.761 .348 & 2,16 & 6,88 & 4.877 .730 & 0,59 & 19,83 & 1.302 \\
\hline Murcia & 832.047 & 30,18 & 1,96 & 1.026 .644 & 14,97 & 20,41 & 866 \\
\hline Navarra & 466.593 & 25,30 & 6,54 & 521.268 & 6,53 & 39,89 & 1.097 \\
\hline País Vasco & 1.867 .287 & 6,75 & 18,48 & 2.129 .197 & 3,03 & 32,42 & 1.342 \\
\hline Rioja & 234.628 & 29,71 & 3,15 & 260.906 & 11,99 & 29,57 & 915 \\
\hline Valencia & 3.078 .095 & 21,07 & 2,83 & 3.786 .546 & 7,13 & 27,65 & 955 \\
\hline
\end{tabular}

Conocidos los valores de los flujos migratorios $T_{i j}$, así como la variable representativa de la fricción espacial, $\mathrm{C}_{\mathrm{ij}}$, y los factores de atracción y rechazo, $V_{i}$ y $W_{i}$, el problema que se plantea es el de calibrar el modelo, obteniendo los valores de los parámetros $\alpha, \beta, \gamma$ que mejor se ajustan al tipo de relación prevista.

La transformación del modelo descrito en una relación lineal permite calibrar el modelo mediante la aplicación de la técnica de regresión múltiple. Esta circunstancia se logra con facilidad al tomar logaritmos (neperianos) en ambos miembros de la ecuación del modelo:

$$
\ln T_{i j}=\ln k+\alpha \ln v_{i}+\beta \ln w_{j}-\gamma \ln c_{i j}
$$

La respuesta de la información empírica al modelo lineal de referencia, en un caso concreto de análisis, constituye una buena prueba de la 
capacidad explicativa del modelo de interacción general. Asimismo, la comparación de los valores obtenidos por los distintos parámetros en dos momentos de tiempo diferentes, así como su nivel de ajuste al modelo, permite extraer o deducir conclusiones sobre la variación de la influencia de los diversos factores, en un estudio evolutivo del fenómeno migratorio.

La aplicación del modelo "no restrictivo» al análisis de la realidad española la hemos realizado en tres fases:

a) Introduciendo las variables determinantes del proceso migratorio, de forma progresiva, con el objeto de analizar individualmente la influencia de cada uno de los factores (año 1990).

b) Una vez definido el modelo general, lo hemos aplicado individualmente a cada una de las comunidades autónomas (tanto como lugar de origen, como de destino de los flujos migratorios).

c) Finalmente, hemos comparado los valores relativos al modelo global, durante los años 1970 y 1990, con la finalidad de obtener una visión dinámica del proceso migratorio.

2.2.1. Influencia de los factores que condicionan la interacción espacial, relativa a un momento concreto en el tiempo (1990)

La investigación del fenómeno migratorio tiene una larga historia, que se remonta al siglo pasado (RAVENSTEIN, E. G., 1885 y 1889). Los principales presupuestos de esta temprana investigación condujeron al modelo gravitatorio de Newton:

$$
T_{i j}=k \frac{P_{i} P_{j}}{d_{i j}^{2}}
$$

donde $P_{i}$ y $P_{j}$ representan los volumenes de población de los lugares de origen y destino, $\mathrm{d}_{\mathrm{ij}}$ la distancia relativa entre ambos y $T_{i j}$ el flujo migratorio resultante.

Tomando como punto de referencia este primitivo planteamiento, hemos comenzado aplicando el modelo de interacción espacial a las tres variables descritas. Los resultados vienen recogidos en el cuadro VI. El nivel de significación es muy elevado para todas las variables (test de la F de Snedecor), superando ampliamente el $99 \%$, lo que da idea de la estrecha relación existente entre el modelo teórico y la información empírica. 
CUADRO VI. AJUSTE DEL MODELO DE INTERACCIÓN ESPACIAL, MEDIANTE LA TÉCNICA DE REGRESIÓN MÚLTIPLE (PASO A)

\begin{tabular}{|c|c|c|c|c|}
\hline $\begin{array}{c}\text { VARIABLE } \\
\text { DEPENDIENTE }\end{array}$ & $\begin{array}{c}\text { CONSTANTE } \\
\text { Y VARIABLES } \\
\text { INDEPENDIENTES }\end{array}$ & TEST $F$ & $\begin{array}{c}\text { VALOR } \\
\text { DEL PARAMETRO }\end{array}$ & $\begin{array}{c}\text { NIVEL } \\
\text { DE } \\
\text { SIGNIFICACIÓN }\end{array}$ \\
\hline $\ln \mathrm{T}_{\mathrm{ij}}$ & $\mathrm{k}$ & - & $-18,0798$ & 0,0000 \\
& $\ln \mathrm{P}_{\mathrm{i}}$ & 251,88 & 0,9209 & 0,0000 \\
& $\ln \mathrm{P}_{\mathrm{i}}$ & 250,16 & 0,9204 & 0,0000 \\
& $\ln \mathrm{d}_{\mathrm{ij}}$ & 25,69 & $-0,3671$ & 0,0000 \\
\hline
\end{tabular}

$R^{2}=0,63766$

La ecuación que relaciona las principales variables del modelo sería, por tanto:

$$
T_{i j}=-18,0798 P_{i}^{0,9209} P_{j}^{0,9204} d_{i j}^{-0,3671}
$$

con un porcentaje explicado de la varianza del $63,77 \%\left(R^{2}=0,63766\right)$.

- El resultado del calibrado de la variable distancia " $\mathrm{d}_{i j}$ " puede ser matizado, si introducimos una nueva variable, representativa de la contigüidad geográfica. Los resultados de diversas investigaciones han permitido demostrar la importancia decisiva de este factor en el incremento relativo de los flujos migratorios. Ello significa, que los movimientos migratorios entre Aragón y Cataluña o entre Castilla la Mancha y Madrid, por poner un ejemplo, se verían incrementados respecto a los correspondientes al valor de la distancia relativa entre ambos, expresada, únicamente, en kilómetros.

La medida de la contigüidad se realiza introduciendo en el análisis una nueva variable, dicotómica, cuyos valores serian únicamente 0 y 1 , según que las unidades territoriales fueran contiguas o no. Las experiencias desarrolladas en este terreno de la investigación aconsejan el empleo de la relación funcional exponencial, en el lugar de la potencial, por lo que el modelo de la interacción espacial se convertiría en:

$$
T_{i j}=K P_{i}^{\alpha} P_{j}^{\beta} d_{i j}^{-\gamma} e^{\lambda b_{i j}}
$$

Los resultados de calibrar el modelo con la información correspondiente a 1990, en el mismo nivel de desagregación espacial, muestran, por una parte, el incremento relativo de la varianza explicada por el modelo de regresión (el $73,11 \%$ frente al $63,77 \%$ ) y, por otra, el casi nulo nivel de significación de la distancia frente a la contigüidad geográfica (en la primera, el nivel de significación estadística la hace ser totalmente rechazable). 
CUADRO VII. AJUSTE DEL MODELO DE INTERACCIÓN ESPACIAL, MEDIANTE LA TÉCNICA DE LA REGRESIÓN MÚLTIPLE (PASO B)

\begin{tabular}{|c|c|c|c|c|}
\hline $\begin{array}{c}\text { VARIABLE } \\
\text { DEPENDIENTE }\end{array}$ & $\begin{array}{c}\text { CONSTANTE } \\
\text { Y VARIABLES } \\
\text { INDEPENDIENTES }\end{array}$ & TEST $F$ & $\begin{array}{c}\text { VALOR } \\
\text { DEL PARÁMETRO }\end{array}$ & $\begin{array}{c}\text { NIVEL } \\
\text { DE } \\
\text { SIGNIFICACIÓN }\end{array}$ \\
\hline $\ln \mathrm{T}_{\mathrm{ij}}$ & $\mathrm{k}$ & - & $-19,8169$ & 0,0000 \\
& $\ln \mathrm{P}_{\mathrm{i}}$ & 306,66 & 0,8811 & 0,0000 \\
& $\ln \mathrm{P}_{i}$ & 306,31 & 0,8807 & 0,0000 \\
& $\mathrm{~b}_{\mathrm{ij}}$ & 92,79 & 1,2437 & 0,0000 \\
& $\ln \mathrm{d}_{\mathrm{ij}}$ & 0,47 & 0,0524 & 0,4916 \\
\hline
\end{tabular}

$\mathrm{R}^{2}=0,7311$

- Finalmente, hemos introducido en el análisis los factores que podrían ser determinantes, por su capacidad de atracción, desde el lugar de destino (el desarrollo económico, medido por el porcentaje de población industrial $\mathrm{l}_{i}$ y el nivel de los salarios $S_{j}$ ) o de rechazo desde el lugar de origen (el bajo nivel de vida, medido por el porcentaje de población agraria $A_{j}$ ). Los resultados del cuadro VIII reflejan la escasa representatividad de todos ellos y su reducida contribución al aumento de la varianza explicada (del 73,11 al 75,38\%). Únicamente, el factor que representa al medio rural refleja un aceptable grado de significación estadistica.

CUADRO VIII. AJUSTE DEL MODELO DE INTERACCIÓN ESPACIAL, MEDIANTE LA TÉCNICA DE LA REGRESIÓN MÚLTIPLE (PASO C)

(AÑO 1990)

\begin{tabular}{|c|c|c|c|c|}
\hline $\begin{array}{c}\text { VARIABLE } \\
\text { DEPPENDIENTE }\end{array}$ & $\begin{array}{c}\text { CONSTANTE } \\
\text { Y VARIABLES } \\
\text { INDEPENDIENTES }\end{array}$ & TEST $\mathrm{F}$ & $\begin{array}{c}\text { VALOR } \\
\text { DEL PARAMETRO }\end{array}$ & $\begin{array}{c}\text { NIVEL } \\
\text { DE } \\
\text { SIGNIFICACIÓN }\end{array}$ \\
\hline $\ln \mathrm{T}_{\mathrm{ij}}$ & $\mathrm{k}$ & - & $-22,8957$ & 0,0000 \\
& $\ln \mathrm{P}_{\mathrm{i}}$ & 64,08 & 0,8377 & 0,0000 \\
& $\ln \mathrm{P}_{\mathrm{i}}$ & 248,68 & 0,8091 & 0,0000 \\
& $\mathrm{~b}_{\mathrm{ij}}$ & 109,11 & 1,3743 & 0,0000 \\
& $\ln \mathrm{A}_{\mathrm{i}}$ & 19,99 & $-0,2296$ & 0,0000 \\
& $\ln \mathrm{S}_{\mathrm{j}}$ & 4,04 & 0,7679 & 0,0455 \\
& $\ln \mathrm{d}_{\mathrm{ij}}$ & 2,12 & 0,1269 & 0,1457 \\
& $\ln \mathrm{I}_{\mathrm{i}}$ & 2,03 & $-0,1973$ & 0,1552 \\
\hline
\end{tabular}

$\mathrm{R}^{2}=0,7538$ 
La representación gráfica de los valores observados en la realidad y los valores predichos por la ecuación de regresión (figura 2) muestra el excelente nivel de ajuste logrado por el calibrado del modelo teórico.

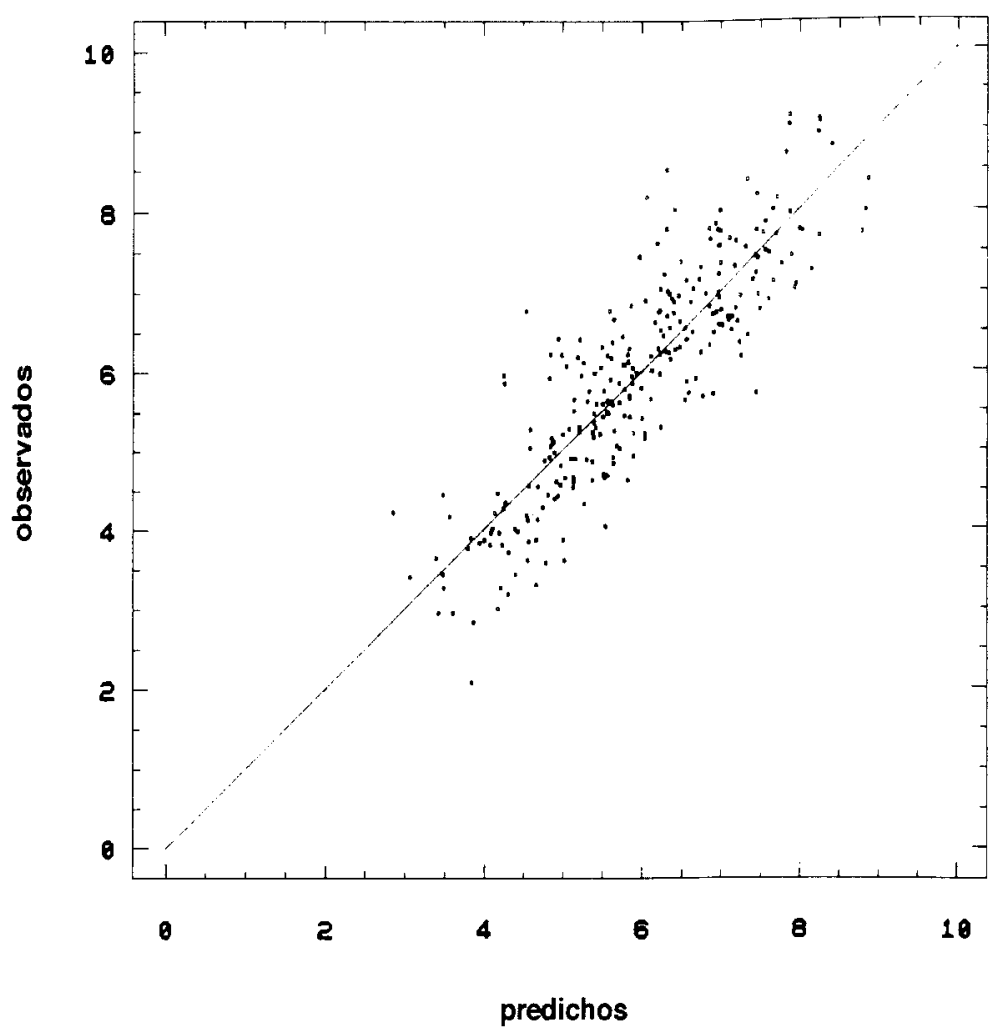

Fig. 2. Representación gráfica de los valores obsenvados y predichos por el análisis de la regresión.

Como resumen general, podemos señalar, que el modelo de interacción espacial es capaz de explicar, en un porcentaje muy significativo, los flujos migratorios habidos en el año 1990 entre las comunidades autónomas españolas. La jerarquía urbana, medida por el volumen de población $\left(P_{i}\right.$ y $\left.P_{j}\right)$, aparece como el factor determinante de los movimientos migratorios interregionales, siendo la contigüidad geográfica $\left(b_{i j}\right)$ mucho más decisiva que la distancia euclidea $\left(\mathrm{d}_{\mathrm{ij}}\right)$. 


\subsubsection{Análisis diferencial por territorios}

En lugar de calibrar el modelo para el total de las relaciones de migración entre cada dos unidades territoriales (272 valores, resultantes de los flujos migratorios de cada comunidad autónoma con las demás, $17 \times 16)$, puede éste ser aplicado, exclusivamente, en el territorio de cada unidad regional. Obtenemos, así, una información más detallada de la influencia de los diversos factores causantes de las migraciones, diferenciada geográficamente.

El estudio puede realizarse desde la doble perspectiva de considerar cada comunidad autónoma como lugar de origen o de destino de la migración (cuadros IX y X). Para facilitar la lectura de los resultados, reflejamos el nivel de significación de cada parámetro de forma cualitativa. Indicamos con el signo más $(+)$, si el test de Snedecor supera el valor de 4 y con el doble signo positivo $(++)$, si el valor es superior a 10.

\section{CUADRO IX. NIVEL DE SIGNIFICACIÓN DE LOS PARÁMETROS REPRESENTATIVOS DE LOS PRINCIPALES FACTORES MIGRATORIOS, POR CADA LUGAR DE ORIGEN (1990)}

\begin{tabular}{|c|c|c|c|c|c|c|}
\hline \multicolumn{7}{|c|}{ VARIABLES } \\
\hline $\begin{array}{c}\text { ÁMBITO } \\
\text { TERRITORIAL }\end{array}$ & $P_{1}$ & $d_{i 1}$ & $b_{n}$ & $\mathrm{~s}_{1}$ & i & $\mathrm{R}^{2}$ \\
\hline Andalucía & ++ & & & & & 54,22 \\
\hline Aragón & ++ & & ++ & + & & 82,60 \\
\hline Asturias & $+t$ & & $+t$ & + & & 89,55 \\
\hline Baleares & ++ & + & & & + & 94,50 \\
\hline Canarias & ++ & & & & + & 92,03 \\
\hline Cantabria & + & & + & & & 79,10 \\
\hline Castilla-León & ++ & & + & ++ & & 86,54 \\
\hline Castilla-La Mancha & ++ & & & & & 79,39 \\
\hline Cataluña & ++ & & & & + & 78,56 \\
\hline Extremadura & + & & & + & & 77,40 \\
\hline Galicia & ++ & + & ++ & ++ & & 91,64 \\
\hline Madrid & ++ & & + & & + & 94,41 \\
\hline Murcia & ++ & & & & & 87,00 \\
\hline Navarra & ++ & & ++ & & & 82,33 \\
\hline País Vasco & ++ & & + & & + & 79,32 \\
\hline Rioja & + & & ++ & & & 71,12 \\
\hline Valencia & ++ & + & + & & $+t$ & 91,43 \\
\hline
\end{tabular}


CUADRO X. NIVEL DE SIGNIFICACIÓN DE LOS PARÁMETROS DE LOS PRINCIPALES FACTORES MIGRATORIOS, PARA CADA LUGAR DE DESTINO (1990)

\begin{tabular}{|c|c|c|c|c|c|}
\hline \multicolumn{6}{|c|}{ VARIABLES } \\
\hline $\begin{array}{c}\text { AMBITO } \\
\text { TERRITORIAL }\end{array}$ & $P_{1}$ & $d_{i j}$ & $b_{i j}$ & $A_{i}$ & $\mathrm{R}^{2}$ \\
\hline Andalucía & ++ & & + & + & 80,73 \\
\hline Aragón & ++ & & + & & 80,82 \\
\hline Asturias & ++ & & ++ & + & 89,31 \\
\hline Baleares & ++ & & n.a. & & 88,80 \\
\hline Canarias & ++ & & n.a. & & 84,49 \\
\hline Cantabria & ++ & & ++ & + & 87,97 \\
\hline Castilla-León & ++ & + & ++ & ++ & 88,21 \\
\hline Castilla-La Mancha & ++ & + & ++ & ++ & 88,15 \\
\hline Cataluña & ++ & & + & & 82,15 \\
\hline Extremadura & ++ & & + & ++ & 88,14 \\
\hline Galicia & + & & + & + & 70,77 \\
\hline Madrid & ++ & & ++ & & 88,05 \\
\hline Murcia & ++ & & ++ & + & 92,42 \\
\hline Navarra & ++ & & ++ & & 79,03 \\
\hline País Vasco & ++ & & ++ & & 63,18 \\
\hline Rioja & ++ & & ++ & & 83,28 \\
\hline Valencia & ++ & & ++ & & 83,48 \\
\hline
\end{tabular}

\section{n.a. $=$ no aplicable}

Las tendencias generales se mantienen en cada territorio individualizado. El volumen poblacional y la contigüidad geográfica aparecen como los factores de mayor peso, frente a la débil influencia del resto. Únicamente, en las regiones más deprimidas (Castilla-León, Castilla-La Mancha, Extremadura, Galicia, etc.) el componente agrario desempeña un papel explicativo aceptable. El resto de los factores muestra su carácter espureo.

\subsubsection{Análisis dinámico del proceso migratorio.}

La comparación de los resultados obtenidos, en dos momentos contrastados en el tiempo (años 1970 y 1990), permite deducir una serie de interesantes conclusiones (cuadros VIII y XI). 
En primer lugar, conviene destacar el elevado grado de ajuste al modelo teórico, en ambos casos, con un porcentaje de la varianza explicada que supera el $70 \%$. Sin embargo, conviene realizar algunas matizaciones:

- La influencia del factor demográfico resulta decisiva en la construcción del modelo interactivo. Incluso, ha ido en aumento con el paso del tiempo. En 1970, el porcentaje de la varianza explicado por la jerarquía poblacional (volúmenes de población de los lugares de origen y destino) era de $51,48 \%$, valor que se incrementó, en 1990 , al $60,29 \%$.

CUADRO XI. AJUSTE DEL MODELO DE INTERACCIÓN ESPACIAL, MEDIANTE LA TÉCNICA DE LA REGRESIÓN MÚLTIPLE (AÑO 1970)

\begin{tabular}{|c|c|c|r|c|}
\hline $\begin{array}{c}\text { VARIABLE } \\
\text { DEPENDIENTE }\end{array}$ & $\begin{array}{c}\text { CONSTANTE } \\
\text { Y VARIABLES } \\
\text { INDEPENDIENTES }\end{array}$ & TEST $F$ & $\begin{array}{c}\text { VALOR } \\
\text { DEL PARÁMETRO }\end{array}$ & $\begin{array}{c}\text { NIVEL } \\
\text { DE } \\
\text { SIGNIFICACIÓN }\end{array}$ \\
\hline $\ln \mathrm{T}_{\mathrm{ij}}$ & $\mathrm{k}$ & - & $-23,6889$ & 0,0000 \\
& $\ln \mathrm{P}_{\mathrm{i}}$ & 195,12 & 0,9998 & 0,0000 \\
& $\ln \mathrm{P}_{\mathrm{i}}$ & 252,84 & 1,1153 & 0,0000 \\
& $\mathrm{~b}_{\mathrm{ij}}$ & 36,88 & 1,1185 & 0,0000 \\
& $\ln \mathrm{A}_{\mathrm{i}}$ & 13,50 & 0,0167 & 0,0003 \\
& $\ln \mathrm{d}_{\mathrm{ij}}$ & 12,49 & $-0,3957$ & 0,0005 \\
& $\ln \mathrm{I}_{\mathrm{j}}$ & 27,41 & 0,0798 & 0,0000 \\
\hline
\end{tabular}

$$
R^{2}=0,7024
$$

- El factor distancia ha ido perdiendo importancia durante los últimos años. Comparando las cifras correspondientes a los años 1970 y 1990, la participación relativa de los factores distancia y contigüidad ha seguido caminos divergentes.

- Finalmente, los factores derivados del poder de atracción o rechazo de los elementos interactivos del proceso migratorio han evolucionado de forma diferenciada. Así, mientras que el factor de impulsión, medido por el porcentaje de población agraria, ha incrementado ligeramente su nivel de significación, los factores de atracción tradicionales derivados de la industria han desaparecido prácticamente. Resulta revelador al respecto la importancia de la industria como agente desencadenante de los movimientos migratorios en los años del desarrollismo y su prácticamente nula participación actual.

A modo de resumen, podemos señalar, que el modelo de interacción espacial muestra en gran medida la estabilidad de los factores determinantes del proceso migratorio regional español. La distancia, como elemento de 
fricción espacial retardador de los movimientos migratorios, ha ido perdiendo importancia con el paso de los años. Por contra, es preciso destacar la decisiva contribución de la contigüidad geográfica como factor explicativo de los desplazamientos de menor alcance.

Finalmente, algunos factores de rechazo tradicionales, como el carácter agrario de una región, han invertido su tendencia (observar el valor de su signo), mientras que otros factores de atracción, como el nivel de desarrollo industrial, han desaparecido prácticamente del horizonte de los factores determinantes de los flujos de migración.

\subsection{La aplicación del modelo restrictivo}

La forma general del modelo restrictivo de origen:

$$
\begin{gathered}
T_{i j}=A_{i} O_{i} \exp \left(-\gamma d_{i j}\right) \\
\text { siendo } A_{i}=\left[\sum_{i} \exp \left(-\gamma d_{i j}\right)\right]^{-1}
\end{gathered}
$$

puede también expresarse de la siguiente manera (FOTHERINGHAM, A. S., y O'KeLLY, M. E., 1987):

$$
T_{i j}=\frac{O_{i} \exp \left[\sum \alpha_{h} f_{h}\left(X_{i j h}\right)\right]}{\sum_{k} \exp \left[\sum_{h} \alpha_{h} f_{h}\left(X_{i j h}\right)\right]}
$$

donde $X_{i i h}$ es la variable explicativa $h$ y $f_{h}$ la forma funcional de dependencia en el modelo.

La ecuación puede ser transformada de manera que sea más fácil de calibrar el modelo:

$$
\ln T_{i j}-\frac{1}{n} \sum_{i} \ln T_{i j}=\sum_{h} \alpha_{h} f_{h}\left(X_{i j h}\right)-\frac{1}{n} \sum_{n} \alpha_{h} \sum_{i} f_{h}\left(X_{i j h}\right)
$$

De acuerdo con ello definimos las siguientes variables, deducidas de las anteriores:

$$
T_{i j}^{*}=\ln T_{i j}-\frac{1}{n} \sum_{i} \ln T_{i j}
$$




$$
\begin{aligned}
& d_{i j}^{*}=\ln d_{i j}-\frac{1}{n} \sum_{i} \ln d_{i j} \\
& b_{i j}^{*}=C_{i j}-\frac{1}{n} \sum_{i} C_{i j} \\
& A_{i}^{*}=\ln A_{i}-\frac{1}{n} \sum_{i} \ln A_{i} \\
& P_{i}^{*}=\ln P_{i}-\frac{1}{n} \sum_{i} \ln P_{i}
\end{aligned}
$$

El resultado de calibrar el modelo, mediante el ajuste de la recta de regresión viene recogido en el cuadro XII.

CUADRO XII. AJUSTE DEL MODELO RESTRICTIVO DE INTERACCIÓN ESPACIAL MEDIANTE LA TÉCNICA DE REGRESIÓN MÚLTIPLE (AÑO 1990)

\begin{tabular}{|c|c|c|c|c|}
\hline $\begin{array}{c}\text { VARIABLE } \\
\text { DEPENDIENTE }\end{array}$ & $\begin{array}{c}\text { CONSTANTE } \\
\text { Y VARIABLES } \\
\text { INDEPENDIENTES }\end{array}$ & TEST F & $\begin{array}{c}\text { VALOR } \\
\text { DEL PARÁMETRO }\end{array}$ & $\begin{array}{c}\text { NIVEL } \\
\text { DE } \\
\text { SIGNIFICACIÓN }\end{array}$ \\
\hline$T^{*}{ }_{i j}$ & $\mathrm{k}$ & - & $1,3403 \mathrm{E}-6$ & 0,0000 \\
& $\mathrm{P}^{*}$ & 337,46 & 0,8100 & 0,0000 \\
& $\mathrm{~d}^{*}{ }^{i}$ & 0,76 & 0,0711 & 0,3821 \\
& $\mathrm{~b}^{{ }^{*}}$ & 141,61 & 1,3908 & 0,0000 \\
& $\mathrm{~A}^{*}{ }_{\mathrm{i}}$ & 30,08 & $-0,2424$ & 0,0000 \\
\hline
\end{tabular}

Si comparamos los resultados con los correspondientes al modelo no restrictivo, podemos observar un mayor nivel de significación en el modelo de restricción de origen (cuadro XIII).

CUADRO XIII. CONTRASTE DE LOS NIVELES DE SIGNIFICACIÓN DE LOS MODELOS NO RESTRICTIVO Y RESTRICTIVO DE ORIGEN

\begin{tabular}{|c|c|c|}
\hline VARIABLES & $\begin{array}{c}\text { MODELO } \\
\text { RESTRICTIV } \\
\text { (VALOR F) }\end{array}$ & $\begin{array}{c}\text { MODELO } \\
\text { RESTRICTIVO } \\
\text { DE ORIGEN } \\
\text { (VALOR F) }\end{array}$ \\
\hline $\mathrm{P}_{i}$ & 250,89 & 337,46 \\
$\mathrm{~d}_{\mathrm{ij}}$ & 2,57 & 0,77 \\
$\mathrm{~b}_{i j}$ & 112,23 & 141,61 \\
$\mathrm{~A}_{i}$ & 322,77 & - \\
$\mathrm{P}_{i}$ & 19,54 & 30,08 \\
\hline
\end{tabular}




\section{CONCLUSIONES}

Los modelos de interacción espacial han demostrado que constituyen un excelente marco teórico de referencia, desde el que plantear $-\mathrm{y}$ resolver- alguna de las problemáticas que afectan a la ciencia geográfica y regional. Desde esta perspectiva, el estudio de los cambios permanentes del lugar de residencia, a diferentes escalas de análisis -internacional, interregional e intraurbana - supone una interesante herramienta algebraica con la que deducir los determinantes básicos de los modelos migratorios de base territorial, relacionando factores de variada indole y contenido: fricción espacial, jerarquía poblacional y variables capaces de medir los desequilibrios regionales que desencadenan los correspondientes flujos migratorios compensatorios.

La aplicación de alguno de los modelos de interrelación espacial (modelos «no restrictivo» y «de restricción de origen»), al análisis de los movimientos migratorios españoles a escala regional, nos ha permitido extraer algunas interesantes conclusiones sobre las tendencias migratorias de nuestro país. Así, el estudio comparativo de los flujos migratorios, entre los territorios de las comunidades autónomas, correspondientes a los años 1970 y 1990, hace posible deducir, con carácter general, que:

- La influencia de la distancia, como factor determinante de las magnitudes migratorias, ha ido disminuyendo progresivamente su importancia, hasta resultar una variable no significativa en este particular proceso de movimiento espacial de la población. Este resultado resulta acorde con la homogeneización y contracción del espacio, fruto del desarrollo alcanzado por los medios de transporte en los países desarrollados.

Sin embargo, la contigüidad geográfica desempeña un papel relevante al multiplicar el efecto migratorio en distancias más reducidas. Conviene, por tanto, distinguir dos tipos de distancias en el proceso de toma de decisión de los lugares de destino de la migración, con un comportamiento claramente diferenciado.

- El volumen de población de los lugares de origen y destino constituyen los factores más estables y definitivos en la explicación de las causas de la migración. Posiblemente, un estudio más detallado de las características demográficas de la población que emigra: edad, sexo, nivel social, etc., reduciria sensiblemente la participación del tamaño de población, como variable indiscriminada, en beneficio de variables más definitivas sobre los motivos de la emi- 
gración. En este sentido, el estudio está abierto a la definición de nuevas variables que reflejen más adecuadamente las características de la oferta (porcentaje de población joven, según sexo y clase social).

- Finalmente, resulta interesante destacar la inversión de tendencia que ha tenido lugar en diversos factores, considerados tradicionales, en el desencadenamiento de los flujos de migración. El efecto multiplicador de la industria, como factor operativo del desarrollo regional, ha disminuido sensiblemente, de manera que los resultados del año 1990 reflejan su nula capacidad de significación. Esta realidad se muestra en consonancia con las nuevas tendencias locacionales de la industria moderna, de mayor nivel de dispersión por el territorio, y menor capacidad de creación de empleo, lo que ha hecho disminuir su potencialidad de atracción. Por su parte, la capacidad de las áreas predominantemente agrarias de impulsar los movimientos de emigración ha desaparecido e incluso invertido su sentido. Este hecho queda de manifiesto en el cambio de signo de los parámetros que estiman el indicador correspondiente al porcentaje de población agraria, de los años 1970 y 1990.

En resumen, el análisis realizado demuestra que las variables de contigüidad espacial y tamaño de población son los factores determinantes que explican la magnitud dominante de los flujos rnigratorios de nuestro país. La significativa variación de la tendencia de las características motrices - de impulsión y atracción - de los movimientos migratorios, respecto al limitado papel de la industria y la inversión de la influencia del medio rural, aunque no definitivos, se muestran en sintonia con los modelos migratorios construidos en los países desarrollados durante los últimos años. 


\section{BIBLIOGRAFÍA}

BATTY, M. (1976): Urban Modelling: Algorithms, Calibrations, Predictions. Cambridge University Press, Cambridge.

BLANCO, C. (1963): «The determinants of interstate population movements», Journal of Regional Science, $n^{\circ} 5$, pp. 77-84.

Campos VenUtTI (1985): "Metrópolis policéntricas y desafío tecnológico" en Metrópolis, Territorio y Crisis. Asamblea de Madrid, Rev. Alfoz, Madrid.

CLARK, G. L., y BALLARD, K. P. (1980): "Modeling out-migration from depressed regions: The significance of origin and destination characteristics». Environment and Planning, no 12, pp. 799-812.

Cordey-HAYES, M., y WILsON, A. G. (1971): "Spatial interaction», SocioEconomic Planning Science, $\mathrm{n}^{\circ}$ 5, pp. 73-95.

DORIGo, G., y TOBLER, W. (1983): «Push-pull migrations laws», Annals of the Association of American Geographers, $n^{\circ} 61$, pp. 228-237.

Evans, R. A. (1969): "The principle of minimum information" Transactions on Reability, $n^{\circ} 7$, pp. 39-61.

FLOWERDEW, R., y SALT, J. (1979): “A method of fitting the gravity model based on the poisson distribution", Journal of Regional Science, $n^{\circ} 13$, pp. 211-231.

FotHeringham, A. S. (1984): "Spatial flows and spatial patterns", Environment and Planning, $\mathrm{n}^{\circ} 16$, pp. 529-543.

Fotheringham, A. S., y O'KelLy, M. E. (1989): Spatial Interaction Models: Formulations and Calibrations, Kubler Academia Publishers, Netherlands. 
HAYNeS, K. E., y Fotheringham, A. S. (1984): Gravity and spatial interaction models, Sage, N. York.

LOVETT, A. A.; WHYTE, I.D., y WHYTE, K. A. (1985): “Poisson regresion analysis and migration fields", Transactions of the Institute of British Geographers, $\mathrm{n}^{\circ} 10$, pp. 317-332.

Méndez, R., y Caravaca, I. (1993): Procesos de reestructuración industrial en las aglomeraciones metropolitanas españolas, M.O.P.T., Madrid.

Murcia, E. (1979): Geografía Urbana: Un punto de vista sistémico, Universidad de Oviedo, Oviedo.

Quefo Castanys, D. (1985): «Experiencias y transformaciones territoriales e institucionales en España" en Metrópolis, Territorio y Crisis, Asamblea de Madrid, Rev. Alfoz, Madrid.

RAvenstein, E. G. (1885): "The laws of migration", Journal of the Royal Statistical Society, $n^{\circ} 48$, pp. 167-235.

RAVENSTEIN, E.G. (1889): "The laws of migration", Second paper, Journal of the Royal Statiscal Society, $\mathrm{n}^{\circ} 52$, pp. 241-305.

SASSEN, S. (1991): "Grandes ciudades: Transformaciones económicas y polarización social" en RODRiguez y otros (cord.): Las grandes ciudades: Debates y propuestas, Economistas Libros, Madrid.

SASSEN, S. (1992): “La ciudad global», Alfoz, C.I.D.U.R., Madrid.

SHANNON, C. F. (1948): "A mathematical theory of communication", Bell System Technical Joumal, ${ }^{\circ} 27$, pp. 379-423 y 623-656.

WILson, A. G., y BENNET, R. J. (1987): Mathematical methods in Human Geography and Planning, John Wiley Sons. 